\title{
Periscope
}

to the psychiatrist, who readily grasps the distress of the personality as a whole, but if some one unfamiliar with such states diagnoses a localised lesion in some organ, the attempt to relieve the patient by treating such, whether by operation or otherwise, will result in failure, and the final result of all therapeutic efforts directed towards such supposedly diseased organs, is to increase and intensify the distress of the personality as a whole, who will continue to struggle and seek for relief far and wide, in and out of the medical profession. Such distress varies greatly from one person to another and may be expressed in unusual and unexpected terms, due to the inherent tendencies and life experiences of the sufferer. It is most important to realise that insidious, chemical, and immunological changes may result from continuous anxiety and depression, and thus doubtless favour metabolic diseases as well as infectious invasions. It cannot be too widely recognised that the attempt to explain all the abnormal feelings and complaints of patients by organic lesions is futile, and that incalculable damage may be done by working along such lines. Thoughts and feelings are as much a product of a patient as the gastric secretions or the pulse-rate, and should be carefully studied in a properly detached manner. The average physician and surgeon entirely overlooks and fails to appreciate the real nature and significance of such states.

Lloyd H. Ziegler (Amer. Journ. Psychiat., r929, viii., 849-879).

\section{NOTE ON THE PAIN SENSATIONS WHICH ACCOMPANY DEEP PUNCTURES.}

The sensations experienced from arterial puncture have a definite character which distinguishes them from those produced by venepuncture and those commonly observed when a needle exerts any tension on the deep fascia.

When a needle reaches the wall of an artery, a dull aching sensation is felt which is much less acute than is a simple puncture of the dermis, but the pain is much less bearable. The pain is diffuse, often referred to some other position (usually distal) and the aching sensation is associated with uncontrollable reflex reactions, such as sudden sensation of warmth. The patient may break out into a sweat, may feel cold, faint, or lose consciousness, or experience nausea. There is a similarity to the sensations experienced from testicular injuries, though the actual pain is less intense. Sensations accompanying punctures of the brachial artery are less intense than those experienced when the radial artery is pierced. Puncture of the smaller vessels is accompanied by more severe sensations and symptoms than is puncture of the larger vessels. 


\section{Periscope}

The sensations from puncture of a vein are usually those of dermal puncture alone and the vessel puncture gives no sensation. Occasionally a small nerve may be affected, but the sensations, although similar to those of arterial puncture, are much less severe.

Punctures made with a needle at right angles to the surface of the skin are accompanied by a simple pricking sensation of an acute type which subsides at once and is accompanied by a slight local inflammatory reaction. If the needle be introduced tangentially the pain is more irritating but not nauseating, and there is a definite relationship between the severity of the pain and the degree of inflammation. A needle can usually be moved back and forward in the subcutaneous fat with little or no pain, and no unpleasant reflex effect or inflammation. Once the needle is within the muscles, sensations are as a rule absent.

As soon as the deep fascia is reached, sensations similar to those described in relation to arterial puncture are found. They are most marked from the outer aspect of the fascia, and subside once the fascia is punctured. If tension be exerted by the needle on the deep fascia, sensations may continue and may even cause the subject to faint. This may be due to a plexus of arterial vessels just superficial to the fascia, or to a nervous network. The more marked sensations of the smaller vessels may be dependent on their richer nerve supply.

The pain from puncture of deep fascial planes and of small arteries resembles that found in certain forms of neuralgia.

H. C. Bazett and B. M'Glone (Brain, r 928, li., I8-23).

\section{THE SYMPTOMATOLOGY OF TUMOURS OF THE TEMPORAL LOBE.}

The temporo-sphenoidal lobe is too large an area of the brain for lesions, variable in type and extent, to produce any one definite train of symptoms and signs. There is thus no definite temporal lobe syndrome.

The author here analyses the various symptoms and signs in their order of frequency found in thirty-eight tumours of this lobe.

Headache was present in every case. There may be local pain or tenderness, and this may be characteristic, coming on suddenly in sharp agonising paroxysms and disappearing abruptly. It is probably due to local pressure on the trigeminal nerve. Vomiting occurred in 50 per cent. of the cases, and incontinence in 20 per cent.; they are merely a symptom of increased intracranial pressure, and have no localising value. Papillœedema was found in 86 per cent. of cases. In the incipient stages the comparative degree of swelling of the two 